\title{
Integrated Sensor System for DNA Amplification and Separation Based on Thin Film Technology
}

\author{
Francesca Costantini, Giulia Petrucci, Nicola Lovecchio, Marco Nardecchia, Augusto Nascetti, \\ Giampiero de Cesare $^{\circledR}$, Lorena Tedeschi, Claudio Domenici, Albert Ruggi, Pisana Placidi, \\ Andrea Scorzoni, and Domenico Caputo
}

\begin{abstract}
This paper presents the development of a lab-onchip, based on thin-film sensors, suitable for DNA treatments. In particular, the system performs on-chip DNA amplification and separation of double-strand DNA into single-strand DNA, combining a polydimethylsiloxane microfluidic network, thin-film electronic devices, and surface chemistry. Both the analytical procedures rely on the integration on the same glass substrate of thinfilm metal heaters and amorphous silicon temperature sensors to achieve a uniform temperature distribution (within $\pm 1{ }^{\circ} \mathrm{C}$ ) in the heated area and a precise temperature control (within $\pm 0.5^{\circ} \mathrm{C}$ ). The DNA separation also counts on the binding between biotinylated dsDNA and a layer of streptavidin immobilized into a microfluidic channel through polymer-brushes-based layer. This approach results in a fast and low reagents consumption system. The tested DNA treatments can be applied for carrying out the on-chip systematic evolution of ligands by exponential enrichment process, a chemistry technique for the selection of aptamers.
\end{abstract}

Index Terms-Amorphous silicon sensor, lab-on-chip (LoC), systematic evolution of ligands by exponential enrichment (SELEX).

\section{INTRODUCTION}

$\mathbf{L}$ ABS-ON-CHIPS (LoCs) are miniaturized analytical systems, which are able to simplify complex laboratory procedures integrating several biochemical procedures on a single chip. The advantages of LoC include reduced sample and reagent consumption, fast detection times, the possibility

This work was supported in part by the Center for Life Nano Scienze at Sapienza, Istituto Italiano di Tecnologia, Rome, Italy, in part by the Sapienza University Research Project 2015 under Grant C26H15J3PX and in part by the MIUR through the project ARTEMIDE under Gran 20108ZSRTR. Recommended for publication by Associate Editor D. De Venuto upon evaluation of reviewers' comments. (Corresponding author: Domenico Caputo.)

F. Costantini and A. Nascetti are with the School of Aerospace Engineering, Sapienza University of Rome, 00138 Rome, Italy (e-mail: augusto.nascetti@uniroma1.it).

G. Petrucci, N. Lovecchio, M. Nardecchia, G. de Cesare, and D. Caputo are with the Department of Information Engineering, Electronics and Telecommunications, Sapienza University of Rome, 00184 Rome, Italy (e-mail: domenico.caputo@uniroma1.it).

L. Tedeschi and C. Domenici are with the Institute of Clinical Physiology, IFC-CNR, 56124 Pisa, Italy (e-mail: lorena.tedeschi@ifc.cnr.it).

A. Ruggi is with the Department of Chemistry, University of Fribourg, 1700 Fribourg, Switzerland (e-mail: albert.ruggi@unifr.ch).

P. Placidi and A. Scorzoni are with the Department of Engineering, University of Perugia, 06125 Perugia, Italy (e-mail: andrea.scorzoni@unipg.it).

Color versions of one or more of the figures in this paper are available online at http://ieeexplore.ieee.org.

Digital Object Identifier 10.1109/TCPMT.2018.2792907 to perform on-field analysis, and no need for expert operators. Their applications comprise agro-food field, forensic science, and analytical and clinical diagnostics [1].

A basic function of an LoC is the recognition and detection of biomolecules, which is often performed through the antigen-antibody reaction [2], [3]. Some researchers have reported DNA extraction and amplification in microfluidicbased device [4], [5]. Recently, DNA biosensors integrated in LoC devices based on microfluidics were also reported [6].

An alternative approach to antibodies, in biomolecular recognition, is represented by aptamers. Aptamers are artificial single-stranded DNA (ssDNA) or RNA sequences that fold into secondary and tertiary structures allowing their binding to selected targets with extremely high specificity [7], [8]. Due to their chemical structure, aptamers are much easier to be chemically modified with functional groups according to different purposes and are much more stable than antibodies. Furthermore, aptamers are selected in vitro via a combinatorial chemistry technique known as systematic evolution of ligands by exponential enrichment (SELEX) overtaking the use of cell lines and animals as starting stages for the production of antibodies, and permitting their production in large quantity with high purity and lower ingredient costs [9].

The SELEX process starts with the generation of a library with a greater number of random nucleotide sequences $\left(10^{13}-10^{16}\right)$, which are incubated with the desired target molecule under conditions suitable for binding. Next, the unbound ssDNA are partitioned from those specifically bound to the target molecule and are then eluted by the target molecules and amplified by the polymerase chain reaction (PCR). This procedure is reiterated for multiple rounds (typically 8-15) until the bound sequences are enriched. SELEX process requires the use of bulky equipment such as shaker and PCR machines and the use of large amounts of reagents and samples.

Although PCR in LoC devices has been largely reported in the literature [10]-[13], only a few examples of the miniaturized SELEX process were reported [14], [15]. These systems are based on microfluidic chip in which magnetic beads functionalized and located into a microfluidic channel are brought into contact with the target molecule. Other works also show the aptamer selection by coated magnetic nanoparticles, PCR amplification, and double-stranded DNA (dsDNA) denaturation in the same chamber of a single microfluidic chip [16]. 


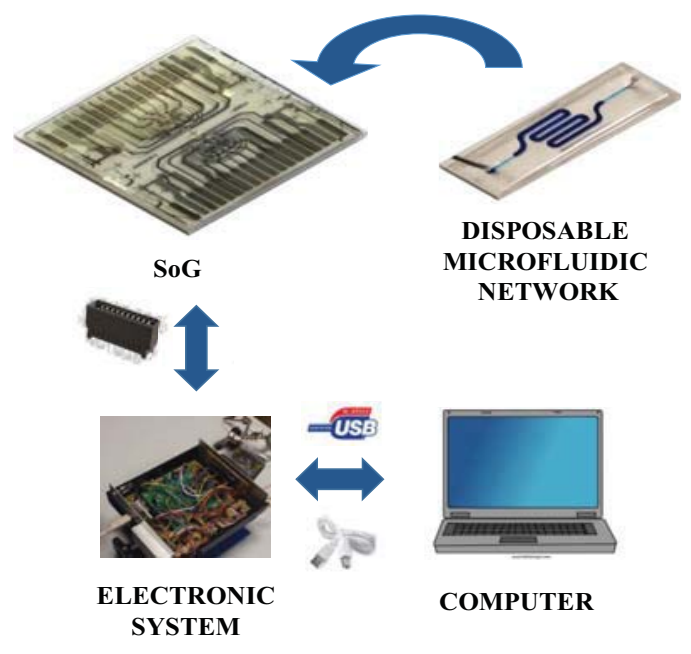

Fig. 1. Schematic of the whole LoC system [12].

These devices combine microfluidic and microelectromechanical system technologies for the miniaturization and automation of the SELEX process with implications in terms of reduction of time and improved efficiency of the whole process. Nevertheless, in these devices, only a few rounds of ligand enrichments were possible, and their applicability for large screening of aptamers seems still very far.

In this paper, we focused on the development of a compact LoC where DNA amplification and dsDNA separation occurred in the same platform. This device can be used in combination with the standard SELEX permitting a quick DNA amplification and separation. The separated ssDNAs could be used for the conventional aptamer selection and in perspective, incorporated within a full on-chip integrated SELEX system. Moreover, the same system could also be used for sensing the presence of pathogens through their DNA amplification, separation, and detection by hybridization with a complementary strand (possibly immobilized in the channel) and final recognition. The compactness and versatility of the device are achieved integrating on the same glass substrate thin-film metal heaters and amorphous silicon (a-Si:H) temperature sensors [17], [18] and coupling the so-obtained systemon-glass (SoG) to a specific polydimethylsiloxane (PDMS) microfluidic network [19].

This paper is organized as follows: Section II reports the details of the LoC structure and fabrication; Section III discusses the experimental results focusing on the DNA amplification through the PCR on chip and on the dsDNA separation through a microfluidic chip functionalized with streptavidin; finally, Section IV draws the conclusions.

\section{LOC STRUCTURE AND FABRICATION}

The whole structure of the developed LoC system is reported in Fig. 1. It includes the following four main parts.

1) SoG hosting on the bottom side of thin-film metal heaters and on the upper side of a-Si:H temperature sensors. In particular, the thin-film metal heaters provide the thermal energy, while the a-Si:H diodes act as temperature sensors for accurate temperature control.
2) A disposable microfluidic network fabricated on another glass and positioned over the SoG during the implementation of the bioanalytical procedures. The designed coupling permits to rapidly substitute the microfluidics when new samples need to be analyzed, avoiding contamination among different samples.

3) An electronic system, electrically connected to the SoG, for the readout of the thin-film temperature sensors and the driving of the heaters.

4) A personal computer that controls the timing and operation of the electronics and displays the results.

The heaters are stacked structures of $\mathrm{Cr} / \mathrm{Al} / \mathrm{Cr}$ layers deposited by vacuum evaporation [18], while the a-Si:H sensors are metal/p-type/intrinsic/n-type/metal structures, deposited by plasma enhanced chemical vapor deposition (PECVD) [20]. Both the heaters and the temperature sensors have been patterned with standard photolithographic processes [21]. Careful attention has been paid to the sequence and recipes of the fabrication steps in order to ensure the compatibility of the different processes and the correct operation of the on-chip sensors. In particular, both the process reliability and the yield increase if the temperature sensors are fabricated before the heaters. This is due to the higher number of photolithographic steps required for the sensor fabrication with respect to the heater.

The fabrication process of the SoG has been then performed with the following steps:

1) cleaning of the glass substrate;

2) fabrication of the a-Si:H sensors through the following:

a) deposition of a $\mathrm{Cr} / \mathrm{Al} / \mathrm{Cr} \quad(300 \dot{\mathrm{A}} / 1500 \dot{\mathrm{A}} / 300 \dot{\mathrm{A}})$ stack acting as bottom electrode and its patterning by photolithography;

b) deposition of the $n-i-p$ a-Si:H layers by PECVD in a three-chamber ultrahigh vacuum system;

c) deposition of a $\mathrm{Cr}$ film $(500 \dot{\mathrm{A}})$ in a Balzers 510 evaporation system;

d) photolithographic patterning of the sensor structure through wet etching of the $\mathrm{Cr}$ layer and reactive ion etching of the a-Si:H layers;

e) deposition of a passivation layer (SU8-3005) and its patterning for the via hole definition over the temperature sensors;

f) deposition by magnetron sputtering of a TiW film $(2000 \dot{A})$ acting as top electrode and its patterning by photolithography;

g) deposition of a passivation layer (SU8-3005) to protect the a-Si:H sensors;

3) fabrication of the thin-film heaters through the following:

a) spin coating of photoresist AZ1518;

b) definition of the geometries of the heaters through UV exposure;

c) development of photoresist in AZ100 remover;

d) thermal vacuum evaporation of three metal $\mathrm{Cr} / \mathrm{Al} / \mathrm{Cr}(1000 \dot{\mathrm{A}} / 6000 \dot{\mathrm{A}} / 1000 \dot{\mathrm{A}})$ stack;

e) liftoff with acetone to remove the metal stack over the cured photoresist; 

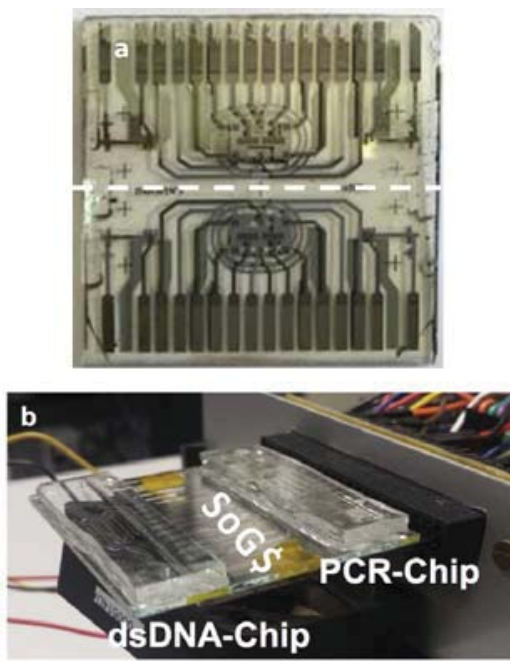

Fig. 2. (a) Fabricated SoG. The lower part of the structure is doubled and mirrored with respect to the white line. (b) dsDNA-chip and PCR-chip (from left to right) positioned on the SoG [12].

f) final passivation of the heaters with SU-8 3005 to protect the structure.

A picture of the fabricated $\mathrm{SoG}$, with the a-Si:H sensors on the top, is reported in Fig. 2(a). Two independent devices are fabricated on a single $50 \mathrm{~mm} \times 50 \mathrm{~mm}$ glass substrate.

The geometry of the central heater has been optimized, using COMSOL multiphysics, to achieve, on the top glass side, a temperature uniformity within $\pm 1{ }^{\circ} \mathrm{C}$, over the area aligned with the heater.

The working principle of the a-Si:H temperature sensors relays on the linear dependence on temperature of the voltage drop across the diode biased with constant forward current [18]. In this paper, a sensitivity of $3.2 \mathrm{mV} /{ }^{\circ} \mathrm{C}$ has been measured at 50-nA forward current bias. A dedicated electronic board drives the heaters and biases and reads out the temperature sensors, providing, at the same time, the temperature control of the PCR process through a proportionalintegrative-derivative (PID) algorithm [22]. The connection of the thin-film devices (heaters and a-Si:H diodes) to the electronic board is implemented by a card edge connector (TE connectivity AMP connector 5-5530843-4).

Two microfluidic chips have been developed: the "amplification chip," (PCR-chip) where the DNA amplification occurs, and the "separation chip," (dsDNA-chip) where the dsDNA is separated into ssDNA.

The PCR-chip is fabricated in PDMS (Sylgard 184, Dow Corning, MI, USA) by soft lithography. PDMS is an elastomer widely used in microfluidics for its good optical transparency, its biocompatibility, and the ease of fabrication. Soft lithography, indeed, allows inexpensive rapid prototyping with low thermal budget. The microfluidics of the PCR-chip has been achieved by bonding together two PDMS layers [17]: the control layer that provides two thermoactuated valves and the flow layer that includes a serpentine-shaped channel for the DNA amplification, an inlet, and an outlet. Fabrication of the thermoactuated valves was achieved mixing the PDMS and the curing agent in the ratio 20:1 and placing the mixture in gentle vacuum at 600 mbar for $30 \mathrm{~min}$ to remove the air bubbles. The PDMS was spun on a previously prepared 50- $\mu \mathrm{m}$-thick SU-8 3050 mold at $500 \mathrm{rpm}$ for $5 \mathrm{~s}$ (acceleration $100 \mathrm{rpm} / \mathrm{s}$ ) and at $1000 \mathrm{rpm}$ for 30s (acceleration of $300 \mathrm{rpm} / \mathrm{s}$ ) in order to obtain a $100-\mu \mathrm{m}$ PDMS thin film. In this way, the valve membrane was $50 \mu \mathrm{m}$ thick. This PDMS layer was partially cured in oven at $85^{\circ} \mathrm{C}$ for $8 \mathrm{~min}$.

The flow channel, instead, has been realized pouring a mixture of PDMS and curing agent in the ratio 10:1 in a 50- $\mu$ m-thick SU8-3050 mold master followed by a partial curing at $80{ }^{\circ} \mathrm{C}$ for $25 \mathrm{~min}$. Also in this case, before pouring, the PDMS and curing agent were placed in gentle vacuum at $600 \mathrm{mbar}$ for $30 \mathrm{~min}$ to remove the air bubbles. The inlet and outlet were made using a 304 SS TiN coated round punch (1.5 in long and featuring a 0.021-in inner diameter and 0.028-in outer diameter) purchased from Syneo LLC (Angleton, TX, USA). Bonding of the PDMS channel with the PDMS valve was achieved by peeling the partially cured PDMS channel from the mold and put onto the partially cured valve layer taking care of the alignment between the channel and the valve. The alignment has been performed under an optical microscope with $10 \times$ magnification. A complete curing of the PDMS network and the bonding of the two layers were achieved in the oven at $60{ }^{\circ} \mathrm{C}$ for $2.5 \mathrm{~h}$. Finally, the whole structure was bonded to a plain glass slide using the transfer bonding technique with uncured PDMS as adhesive [23].

The layout of the PCR module ensures the alignment of the microfluidics with the corresponding electronic devices on the SoG. The dimensions of the microfluidic network determine a useful-PCR volume close to $6 \mu \mathrm{L}$. Prior to use, the PDMS channel was filled with a solution of bovine serum albumin (BSA) $4 \%$ at the flow rate of $25 \mu \mathrm{L} / \mathrm{min}$ for $10 \mathrm{~min}$ and $5 \mu \mathrm{L} / \mathrm{min}$ for $1 \mathrm{~h}$. After rinsing with MilliQ water at $25 \mu \mathrm{L} / \mathrm{min}$ for $10 \mathrm{~min}$, the chip was dried with a stream of nitrogen and kept in the oven at $37{ }^{\circ} \mathrm{C}$ until the channel appeared completely dried.

The dsDNA-chip, made in Sylgard 184, has the same dimensions and shape of the flow layer of the microfluidic PCR module but does not include the control layer. The fabrication starts from the pouring of a mixture of PDMS and curing agent in the ratio 10:1 on the same mold used for the flow layer of the PCR module and proceeds with a complete curing at $100{ }^{\circ} \mathrm{C}$ for $40 \mathrm{~min}$. Subsequently, the inlet and outlet are made. Before bonding, a glass substrate was functionalized with polymer brushes using a previously published procedure [24]-[28].

At this point, the PDMS stub, previously obtained, was bonded to the poly 2-(hydroxyethyl methacrylate) (PHEMA) functionalized with succinic anhydride functionalized glass substrate [23]. After the bonding between the PDMS serpentine channel and the PHEMA-functionalized glass slide, the microfluidic channel was further modified to immobilize streptavidin [7].

The amount of streptavidin immobilized to the brush layer was quantified using a bicinchoninic acid assay [29] protein assay kit (Thermo Scientific). Several standard samples were prepared with a known concentration of BSA and a working reagent. Using these standards, a calibration curve was determined (by plotting the BSA concentration versus 


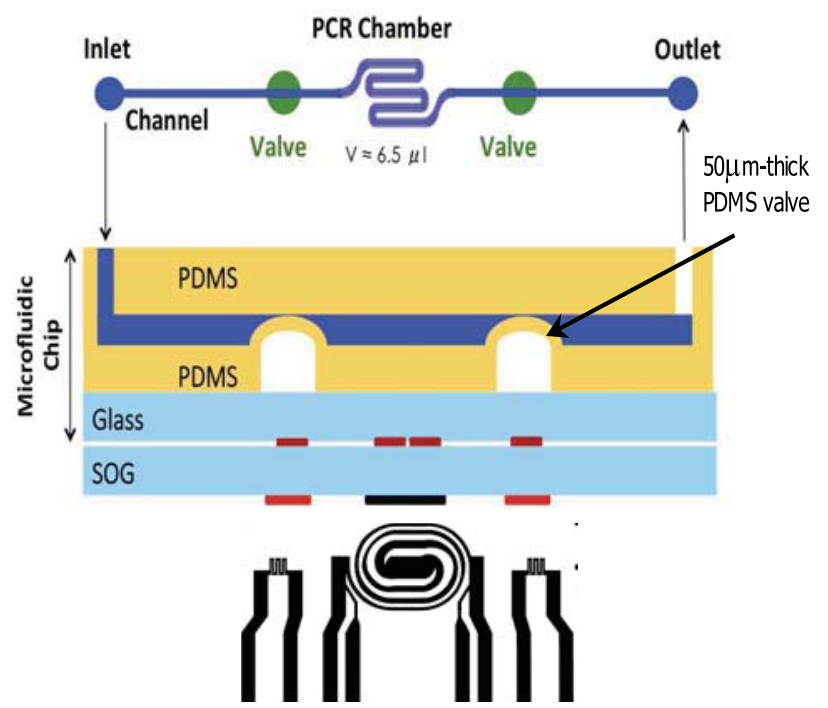

Fig. 3. Schematic of PCR-chip coupled with the SoG. The lower part shows the three heaters (black lines): The central one has a double concentric spiral shape, while the two lateral heaters have a meander shape. The turning ON of the heaters aligned with the valves determines the deflection of the PDMS membrane of the control layer and the subsequent closure of the microfluidic channel.

the absorbance at $562 \mathrm{~nm}$ as measured for each standard sample). The working reagent solution was pumped into the chip and kept inside for $30 \mathrm{~min}$ at room temperature. Subsequently, the chip was rinsed with buffer, and the eluted solution collected with a Hamilton syringe and injected into a flow cell connected by means of two optical fibers (Ocean Optics Inc., The Netherlands), and this cell was connected to a deuterium-halogen light source (DT-Mini-2-GS, Mikropack GmbH, Germany) and a fiber optic spectrometer (HR4000, Ocean Optics Inc., The Netherlands). Subsequently, the absorbance at $562 \mathrm{~nm}$ was measured, and the concentration of the streptavidin was calculated using the calibration curve. The total concentration of streptavidin was found to be $(3.1 \pm 0.46) 10^{-5} \mu \mathrm{g} / \mathrm{mm}^{2}$.

\section{RESUlts AND Discussion}

In order to perform the DNA treatments, the SoG has been coupled to the PCR-chip and to the separation chip as reported in Fig. 2(b). It is worth noting that the fabricated SoG can host both the PCR-chip and the dsDNA-chip.

\section{A. PCR on Chip}

During the DNA amplification, the PCR-chip is placed on the SoG, aligning the valves and the chamber with the heaters (Fig. 3). The two meander-shaped lateral heaters are aligned with the valves, providing the deflection of the $50-\mu \mathrm{m}$ thick PDMS membrane of the control layer (Fig. 3), while the central serpentine-shaped heater, aligned with the PCR chamber, supplies the energy for the DNA thermal treatment.

A PCR reaction mix (20-nM ssDNA template, $1 \mu \mathrm{M}$ of each primer, $0.2-\mathrm{mM}$ deoxynucleoside triphosphates, and $1.5-\mathrm{mM}$ $\mathrm{MgCl}_{2}$ ) was prepared in the reaction buffer and an aliquot was stored as control mix. A Hot Start Taq enzyme (HOT FIREPol DNA Polymerase-Solis BioDyne) was added to the remaining

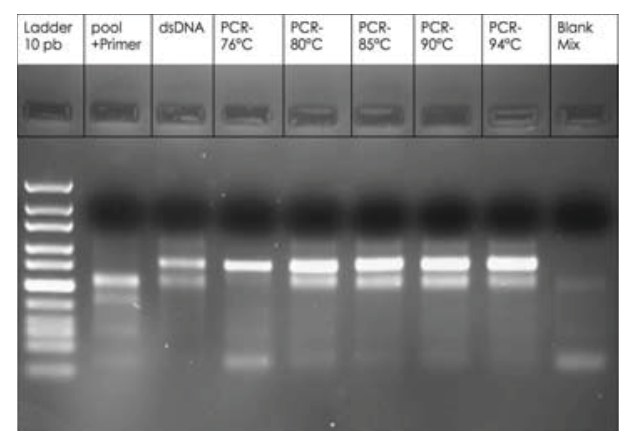

Fig. 4. Gel electrophoresis of dsDNA after the PCR amplification by applying different denaturation temperatures. dsDNA is the product of a PCR reaction conducted under the optimized conditions for this template and primers, used as reference/standard product.

sample to a final concentration of $0.05 \mathrm{U} / \mu \mathrm{L}$. This solution was then inserted in the microfluidic network.

As the channel and the process chamber were filled, the two heaters below the valves were actuated. Applying power to the heaters, the air contained in the valve reservoir heats up and increases its volume creating a pressure that pushes up the PDMS membrane into the channel. As the valves are closed, the thermal procedure can occur by turning on the heater below the PCR chamber.

The temperature control is performed in the PCR process through the PID algorithm implemented by the electronic control board ensuring the absence of overshoots and a deviation from the set-point temperature below $\pm 0.5{ }^{\circ} \mathrm{C}$. The heating rate achieved by the system is $2{ }^{\circ} \mathrm{C} / \mathrm{s}$, while the cooling rate, controlled by a fan driven by the same electronic board, is $1{ }^{\circ} \mathrm{C} / \mathrm{s}$.

Typically, the PCR process is conducted using a threetemperature sequence: melting of the double-stranded DNA (at about $94{ }^{\circ} \mathrm{C}$ ), annealing of the specific primers to their ssDNA template (usually between 50 and $65{ }^{\circ} \mathrm{C}$, depending on the primer sequence and length), and extension of primers by a thermostable polymerase enzyme (Taq polymerase) (near $72 \mathrm{C}$ ). In order to perform PCR in the microfluidic chip, preliminary experiments have been conducted to observe the effect of the temperature variation on the microfluidic chip filled with a buffer solution. Varying the temperature between $55{ }^{\circ} \mathrm{C}$ and $95{ }^{\circ} \mathrm{C}$, bubble formation was observed above $80{ }^{\circ} \mathrm{C}$, causing partial evaporation of the sample and leading to irreproducible values of the amplification yield for samples under the same amplification conditions.

In order to avoid the bubble formation in the chip, the PCR process has been revised and optimized in order to avoid temperatures above $80{ }^{\circ} \mathrm{C}$. A set of experiments has been conducted by varying the temperature of the melting step in the range of $76{ }^{\circ} \mathrm{C}-90{ }^{\circ} \mathrm{C}$ using a standard commercial thermocycler. The gel electrophoresis conducted for each sample showed that the amplicon (Fig. 4) was formed for each selected temperature.

Taking into account these results, the on-chip PCR was conducted using the following procedure: $20 \mathrm{~s}$ at $76{ }^{\circ} \mathrm{C}$ (melting), $60 \mathrm{~s}$ at $62{ }^{\circ} \mathrm{C}$ (annealing), and $30 \mathrm{~s}$ at $72{ }^{\circ} \mathrm{C}$ (elongation). In order to activate the Taq polymerase, the solution containing oligonucleotides, ssDNA and DNA polymerase, has been 


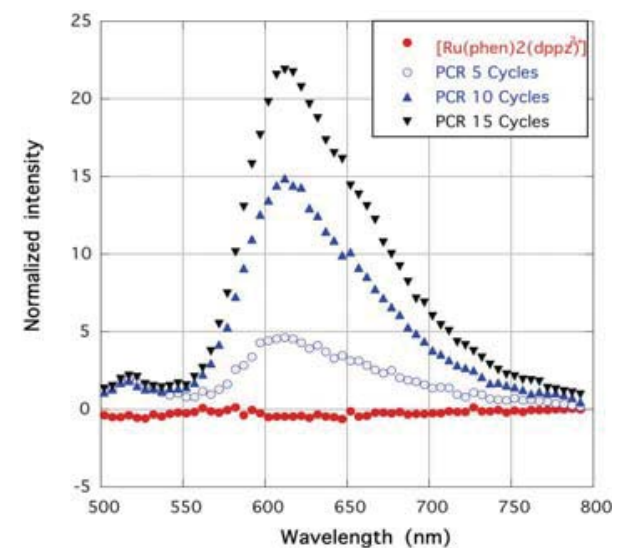

Fig. 5. Normalized emission spectra as a function of the PCR cycles. The normalization has been achieved by subtracting the blank signal.

previously heated at $94{ }^{\circ} \mathrm{C}$ for 15 min off-chip. Subsequently, $30 \mu \mathrm{L}$ of that solution were flowed into the channel using a Hamilton syringe. PCR experiments have been conducted varying the number of thermocycles from 5 to 15 . 15-cycle 15 was also performed using a solution without Taq polymerase (blank).

The yield of the amplification was verified off-chip: The channel was rinsed with phosphate buffer, and the collected sample was lyophilized. Subsequently, $1 \mathrm{~mL}$ of a phosphate buffer solution containing $[\mathrm{Ru}(\mathrm{phen}) 2(\mathrm{dppz})]^{2+} 0.1 \mathrm{mM}$ was added to each sample. $[\mathrm{Ru}(\mathrm{phen}) 2(\mathrm{dppz})]^{2+}$ is a light switch complex [30], which has no luminescence in aqueous solution as the triplet metal to ligand charge transfer excited state is quenched by hydrogen binding between water and the phenazine nitrogen of the ligand. When it binds to dsDNA, the interaction between the ligand and the base pair of duplex nucleic acid protects the phenazine nitrogen from water, leading to an intense emission.

The PCR amplified samples were analyzed by fluorescence spectroscopy. As expected, the analysis showed an increase of the fluorescent signal proportional to the number of cycles performed (Fig. 5). The blank signal showed the same luminescence intensity of the solution containing only $[\operatorname{Ru}(\text { phen }) 2(\mathrm{dppz})]^{2+}$. The samples were also analyzed by gel electrophoresis to verify that the right strand of ssDNA was amplified (Fig. 6). The gel-electrophoresis proves the amplification of the ssDNA to the desired dsDNA; moreover, no amplification was observed for the blank sample, thus confirming the results obtained by fluorescence spectroscopy.

\section{B. dsDNA Separation on Chip}

After DNA amplification, the dsDNA is separated to ssDNA. This module is located in the same platform. The PCR product is taken using a Hamilton syringe and is directly transferred into "dsDNA separation chip." This channel is functionalized with streptavidin and will bind the biotinylated dsDNA. After heating till $95^{\circ} \mathrm{C}$, the dsDNA will be separated and would be possible to recover the ssDNA and possibly use it for conventional aptamer selection for a given target molecule.

This step is usually performed using streptavidin-coated beads through which the dsDNA, having one of the strands

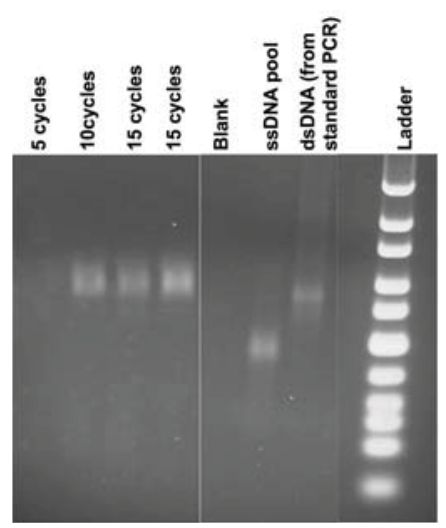

Fig. 6. Gel electrophoresis results showing that the right strand of DNA was amplified. Indeed, the band of the dsDNA amplified with the on-chip PCR has the same position of the band achieved with a standard PCR.

labeled with biotin, is brought into contact. After several washing steps and the elution of the nonbiotinylated strand, the ssDNA is achieved [31], [32].

As reported before, in this paper, the separation of the amplified dsDNA is obtained using a microfluidic chip (dsDNA-chip) functionalized with streptavidin by growing PHEMA polymer brushes on the glass side of the channel.

The dsDNA-chip has the same structure of the PCR chip, i.e., serpentine chamber having $6.5-\mu \mathrm{L}$ volume without the thermoactuated valves, which are not necessary since the dsDNA separation process is occurring in continuous flow. The dsDNA-chip is positioned on the top of the integrated device, aligning the main heater with the serpentine chamber.

The product of the PCR amplification is a dsDNA having a biotinylated reverse primer, thus we tailored that once the dsDNA is immobilized to the streptavidin layer, the increase in temperature up to $95{ }^{\circ} \mathrm{C}$ for a few seconds would recover the nonbiotinylated ssDNA representing the desired sequence to be incubated with the target molecule for the selection of the aptamer. Thus, the amplified dsDNA $(0.6 \mu \mathrm{M})$ was inserted into the dsDNA-chip, by means of a Hamilton syringe, and incubated for $10 \mathrm{~min}$. The channel was rinsed with a phosphate buffer at the flow rate of $15 \mu \mathrm{L} / \mathrm{min}$ for $5 \mathrm{~min}$ for three times to eliminate the unbounded dsDNA. The rinsing buffer was collected after each rinsing step in a vial and named "flow through vials" (FTv). Afterward, a MilliQ water solution was flowed into the channel and a temperature of $95{ }^{\circ} \mathrm{C}$ was set on the main heater of the SoG device.

In this case, the electronic circuit driving the heater and controlling the temperature is based on the variation of the heater resistance with temperature controlled through the analog circuit reported in [33]. The control circuit is based on a single operational amplifier fed with a single supply voltage. The circuit implements a constant resistance control loop [34], [35]. In the circuit, a Darlington bipolar junction transistor (BJT) has been used to bias the microheater, due to the relatively high current needed. An additional BJT limits the maximum current of the heater. In addition, a feedback resistor has been split in two parts in order to accurately define a limiting current for the heater. A voltage divider, implemented 


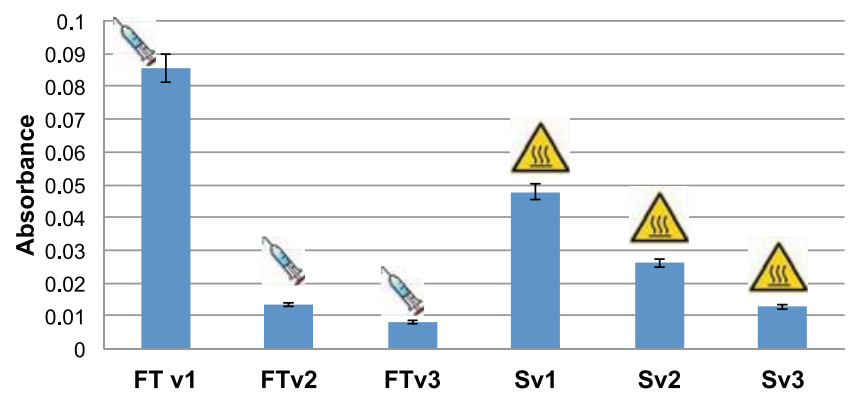

Fig. 7. Analysis by UV-Vis spectroscopy of FTv and Sv samples.

with a digital potentiometer, has been used to change the target temperature of the heater. The maximum achievable heating rate is limited by the maximum power density on the glass that prevents fractures and cracking. In particular, an average heating rate of $7{ }^{\circ} \mathrm{C} / \mathrm{s}$ and a regime temperature ripple of the order of $\pm 0.5^{\circ} \mathrm{C}$ were achieved. An active cooling system was not necessary and was not implemented. The actual value of the final temperature reached by the heater slightly depends on the room temperature since the measured 2-wire heater resistance depends on an effective temperature coefficient of resistance, which encloses the room temperature. However, the regime heater temperature for the dsDNA-chip is not so critical as for the PCR module as it is enough to increase the temperature above the melting point of the dsDNA to achieve the separation of the double strands.

After heating the channel at $95{ }^{\circ} \mathrm{C}$ for $30 \mathrm{~s}$, the heater was turned OFF and, subsequently, the microchannel was rinsed using MilliQ water at the flow rate of $5 \mu \mathrm{L} / \mathrm{min}$ for $2 \mathrm{~min}$ and at $15 \mu \mathrm{L} / \mathrm{min}$ per $5 \mathrm{~min}$. This procedure was repeated thrice and all the samples were collected in three different vials named "sample vials" (Sv). At the temperature of $95{ }^{\circ} \mathrm{C}$, the dsDNA denaturates, allowing the separation of the complementary sequences. The biotinylated strand remains bound to the PHEMA-streptavidin layer, while the other strand is removed by flowing water applied through the channel. In order to verify this hypothesis, all the FTv and Sv samples were lyophilized, and subsequently re-dissolved in MilliQ water and analyzed by UV-vis spectroscopy following the absorption at $260 \mathrm{~nm}$.

Fig. 7 shows that, as expected, the amount of dsDNA in the FTv samples decreases from the FTv1 to FTv3 after the third rinsing step and that some DNA is still present in FTv3. This result suggests that probably some of the dsDNA remains bound onto the PHEMA-streptavidin layer or nonspecifically adsorbed onto the surface of the PDMS channel. The analysis of the Sv samples revealed the presence of DNA in each vial, even though the amount of DNA in Sv3 resulted diminished compared to Sv1. In order to verify whether the DNA collected was dsDNA or ssDNA, the Sv1 was also analyzed by gelelectrophoresis. The analysis revealed that ssDNA was retried by applying the described procedure. This experiment demonstrates the capability of the PHEMA-Streptavidin functionalized chip to separate the dsDNA obtained by PCR.

A device based on this technology was never reported in the literature. This system compared to those described [10], [11] is solely based on surface chemistry functionalization of the microchannel and does not need the magnetic beads to be introduced into the system. Moreover, the developed streptavidin layer can also be used to immobilize a specific biotinylated ssDNA, which may eventually be used for the recognition, by hybridization, of a selected ssDNA for sensing applications.

The use of the proposed dsDNA-chip, compared with the most commonly used laboratory procedure for isolation of ssDNA based on magnetic separation with streptavidin-coated beads, is advantageous in terms of both cost and time. Indeed, in order to perform the separation, the chip procedure requires a few microliters of amplified DNA $(6.5 \mu \mathrm{L})$ versus the $100 \mu \mathrm{L}$ necessary for the laboratory procedure [32]. Moreover, for the dsDNA-chip, the preconditioning steps are not necessary and the separation of the dsDNA occurs in 45 min instead of about $2 \mathrm{~h}$ as for the magnetic beads procedure [31]. Finally, the DNA separation achieved by applying the dsDNA-chip does not need the centrifugation step, as instead required by the beads, avoiding the use of bulky and expensive equipment.

\section{Conclusion}

This paper is focused on the development an LoC aiming to DNA amplification and to separation of dsDNA into ssDNA. The LoC results from the combination of two glass substrates: One is provided with thin-film electronic devices for supplying and controlling the thermal energy, and the other is bonded to a PDMS microfluidic network, where DNA manipulation is performed. In particular, our LoC presents for the first time: 1) a DNA amplification controlled by thin-film metal heaters coupled with a-Si:H temperature sensors, and 2) a DNA separation ruled by the biotin-streptavidin bond, where the streptavidin is immobilized on the glass surface through a PHEMA polymer brush layer.

\section{REFERENCES}

[1] G. Luka et al., "Microfluidics integrated biosensors: A leading technology towards lab-on-a-chip and sensing applications," Sensors, vol. 15, no. 12, pp. 30011-30031, 2015.

[2] A. B. Iliuk, L. Hu, and W. A. Tao, "Aptamer in bioanalytical applications," Anal. Chem., vol. 83, no. 12, pp. 4440-4452, 2011.

[3] D. De Venuto, S. Carrara, and B. Riccò, "Design of an integrated lownoise read-out system for DNA capacitive sensors," Microelectron. J., vol. 40, no. 9, pp. 1358-1365, 2009.

[4] J.-G. Lee, K. H. Cheong, N. Huh, S. Kim, J.-W. Choi, and C. Ko, "Microchip-based one step DNA extraction and real-time PCR in one chamber for rapid pathogen identification," Lab Chip, vol. 6, no. 7, pp. 886-895, 2006.

[5] F.-C. Huang, C.-S. Liao, and G.-B. Lee, "An integrated microfluidic chip for DNA/RNA amplification, electrophoresis separation and online optical detection," Electrophoresis, vol. 27, no. 16, pp. 3297-3305, 2006.

[6] M. I. H. Ansari, S. Hassan, A. Qurashi, and F. A. Khanday, "Microfluidic-integrated DNA nanobiosensors," Biosensors Bioelectron., vol. 85, pp. 247-260, Nov. 2016.

[7] F. Costantini et al., "Aptamer-based sandwich assay for on chip detection of Ochratoxin A by an array of amorphous silicon photosensors," Sens. Actuators B, Chem., vol. 230, pp. 31-39, Jul. 2016.

[8] S. Song, L. Wang, J. Li, J. Zhao, and C. Fan, "Aptamer-based biosensors," TrAC Trend Anal. Chem., vol. 27, no. 2, pp. 108-117, 2008.

[9] M. Darmostuk, S. Rimpelova, H. Gbelcova, and T. Ruml, "Current approaches in SELEX: An update to aptamer selection technology," Biotechnol. Adv., vol. 33, no. 6, pp. 1141-1161, 2015. 
[10] F. Ahmad and S. A. Hashsham, "Miniaturized nucleic acid amplification systems for rapid and point-of-care diagnostics: A review," Anal. Chim. Acta, vol. 733, pp. 1-15, Jul. 2012.

[11] C. D. Ahrberg, B. R. Ilic, A. Manz, and P. Neužil, "Handheld real-time PCR device," Lab Chip, vol. 16, no. 3, pp. 586-592, 2016.

[12] Y. Zhang and P. Ozdemir, "Microfluidic DNA amplification-A review," Anal. Chim. Acta, vol. 638, no. 2, pp. 115-125, 2009.

[13] G. Petrucci et al., "Multifunctional system-on-glass for lab-onchip applications," Biosensors Bioelectron., vol. 93, pp. 315-321, Jul. 2017.

[14] C.-J. Huang, H.-I. Lin, S.-C. Shiesh, and G.-B. Lee, "Integrated microfluidic system for rapid screening of CRP aptamers utilizing systematic evolution of ligands by exponential enrichment (SELEX)," Biosensensors Bioelectron., vol. 25, 7, pp. 1761-1766, 2010.

[15] L.-Y. Hung, C.-H. Wang, K.-F. Hsu, C.-Y. Chou, and G.-B. Lee, "An on-chip Cell-SELEX process for automatic selection of high-affinity aptamers specific to different histologically classified ovarian cancer cells," Lab Chip, vol. 14, no. 20, pp. 4017-4028, 2014

[16] H.-I. Lin, C.-C. Wu, C.-H. Yang, K.-W. Chang, G.-B. Lee, and S.-C. Shiesh, "Selection of aptamers specific for glycated hemoglobin and total hemoglobin using on-chip SELEX," Lab Chip, vol. 15, no. 2, pp. 486-494, 2015.

[17] A. Zahra, R. Scipinotti, D. Caputo, A. Nascetti, and G. De Cesare, "Design and fabrication of microfluidics system integrated with temperature actuated microvalve," Sens. Actuators A, Phys., vol. 236, pp. 206-213, Dec. 2015.

[18] D. Caputo, G. de Cesare, A. Nascetti, and R. Scipinotti, "a-Si:H temperature sensor integrated in a thin film heater," Phys. Status Solidi A, vol. 207, no. 3, pp. 708-711, 2010.

[19] F. Costantini et al., "Lab-on-glass system for DNA treatments," in Proc. 7th Int. Workshop Adv. Sensors Interfaces (IWASI), Jun. 2017, pp. 241-245.

[20] D. Caputo et al., "A systematic investigation of the role of material parameters in metastability of hydrogenated amorphous silicon," J. Non-Cryst. Solids, vol. 170, no. 3, pp. 278-286, 1994.

[21] D. Caputo, M. Ceccarelli, G. De Cesare, A. Nascetti, and R. Scipinotti, "Lab-on-glass system for DNA analysis using thin and thick film technologies," in Proc. Mater. Res. Soc. Symp., vol. 1191. 2009, pp. 53-58.

[22] N. Lovecchio et al., "Thermal control system based on thin film heaters and amorphous silicon diodes," in Proc. 6th IEEE Int. Workshop Adv. Sensors Interfaces (IWASI), Jun. 2015, pp. 277-282.

[23] S. Satyanarayana, R. N. Karnik, and A. Majumdar, "Stamp-andstick room-temperature bonding technique for microdevices,' J. Microelectromech. Syst., vol. 14, no. 2, pp. 392-399, 2005.

[24] F. Costantini et al., "A new nanostructured stationary phase for ultra-thin layer chromatography: A brush-gel polymer film," Nanosci. Nanotechnol. Lett., vol. 5, no. 11, pp. 1155-1163, 2013.

[25] F. Costantini et al., "On-chip detection of multiple serum antibodies against epitopes of celiac disease by an array of amorphous silicon sensors," RSC Adv., vol. 4, no. 4, pp. 2073-2080, 2014

[26] F. Costantini et al., "Lab-on-chip system combining a microfluidicELISA with an array of amorphous silicon photosensors for the detection of celiac disease epitopes," Sens. Biosensing Res., vol. 6, pp. 51-58, Dec. 2015.

[27] R. Salvio and A. Cincotti, "Guanidine based self-assembled monolayers on Au nanoparticles as artificial phosphodiesterases," Rsc Adv., vol. 4 , no. 54, pp. 28678-28682, 2014

[28] D. Caputo et al., "On-chip diagnosis of celiac disease by an amorphous silicon chemiluminescence detector," in Sensors and Microsystems, vol. 268. Cham, Switzerland: Springer, 2014, pp. 183-187.

[29] F. Costantini et al., "Glucose level determination with a multi-enzymatic cascade reaction in a functionalized glass chip," Analyst, vol. 138, no. 17, pp. 5019-5024, 2013

[30] Y. Jiang, X. Fang, and C. Bai, "Signaling aptamer/protein binding by a molecular light switch complex," Anal. Chem., vol. 76, no. 17, pp. 5230-5235, 2004

[31] C. Marimuthu, T.-H. Tang, J. Tominaga, S.-C. Tan, and S. C. B. Gopinath, "Single-stranded DNA (ssDNA) production in DNA aptamer generation," Analyst, vol. 137, no. 6, pp. 1307-1315, 2012 .

[32] M. Svobodová, A. Pinto, P. Nadal, and C. K. O'Sullivan, "Comparison of different methods for generation of single-stranded DNA for SELEX processes," Anal. Bioanal. Chem., vol. 404, no. 3, pp. 835-842, 2012 .
[33] A. Scorzoni, M. Tavernelli, P. Placidi, P. Valigi, and A. Nascetti, "Accurate analog temperature control of a thin film microheater on glass substrate for lab-on-chip applications," in Proc. IEEE SENSORS, Nov. 2014, pp. 1216-1219.

[34] CTSO3-Propane/LPG Data Sheet, First Technol. PLC, Honeywell, Morris Plains, NJ, USA, 2006.

[35] C. Sosna, R. Buchner, and W. Lang, "A temperature compensation circuit for thermal flow sensors operated in constant-temperature-difference mode," IEEE Trans. Instrum. Meas., vol. 59, no. 6, pp. 1715-1721, Jun. 2010.

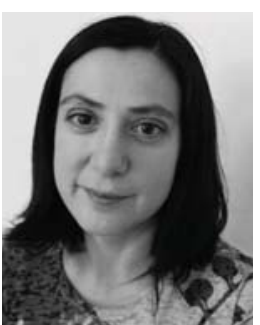

Francesca Costantini received the master's degree in medical chemistry from Bologna University, Bologna, Italy, and the Ph.D. degree in chemistry from the University of Twente, Enschede, The Netherlands, in 2009.

She is currently a Post-Doctoral Researcher with the University of Rome Sapienza, Rome, Italy, where she is involved in the development of microanalytical devices for healthcare and diagnostics. Her current research interests include the immobilization of nanometallic, organic, and enzymatic catalysts in microreactors.

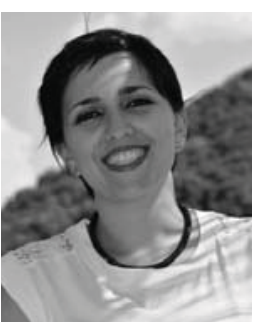

Giulia Petrucci received the master's degree in biomedical engineering and the Ph.D. degree in information and communication technology (ICT-curriculum electronic engineering) from the University of Rome "La Sapienza," Rome, Italy, in 2013 and 2017, respectively.

Her current research interests include the development of lab-on-chip systems integrating microfluidics, photosensors, and thin-film heaters for clinical and diagnostics.

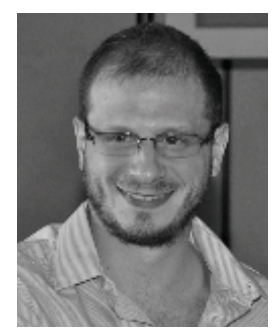

Nicola Lovecchio received the master's degree in electronic engineering from the University of Rome "La Sapienza," Rome, Italy, in 2014, where he is currently pursuing the Ph.D. degree in information and communication technology (ICT-curriculum electronic engineering).

His current research interests include the development of electronic circuits for driving lab-on-chip systems-based electrowetting on dielectric technique for biochemical analysis.

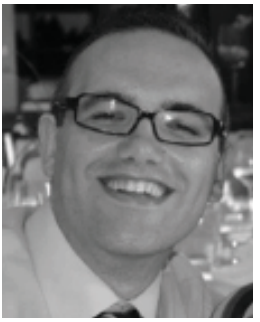

Marco Nardecchia received the master's degree in electronic engineering from the University of Rome "La Sapienza," Rome, Italy, in 2014, where he is currently pursuing the Ph.D. degree in information and communication technology (ICT-curriculum electronic engineering).

His current research interests include the development of lab-on-chip systems integrating microfluidics, photosensors, and thin-film heaters for biochemical analysis and space applications.

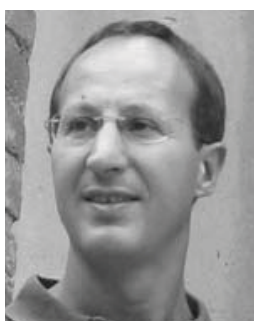

Augusto Nascetti is currently an Associate Professor with the School of Aerospace Engineering, University of Rome "La Sapienza," Rome, Italy. $\mathrm{He}$ is also the Principal Investigator of the project "PLEIADES" dedicated to the design and realization of a new analytical integrated platform for the multiparametric detection of life markers in extraterrestrial environments. His current research interests include electronic systems for radiation detectors and lab-on-chip-systems with integrated amorphous silicon sensors. 


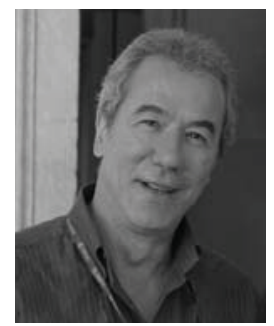

Giampiero de Cesare has been an Associate Professor with the Faculty of Engineering, Rome University "La Sapienza," Rome, Italy, since 1998, where he is currently the Head of the Microelectronic Technology Laboratory, Department of Information Engineering, Electronics and Telecommunications. His current research interests include amorphous silicon technology and its application in thin-film devices for imaging detection and photovoltaic systems and in the development of innovative "lab-on-chip" systems based on a-Si photodiodes, for molecular analysis and food quality control applications.

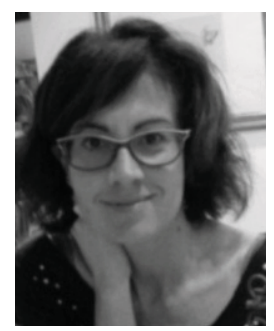

Lorena Tedeschi received the Laurea degree in pharmaceutical chemistry and technology and the Ph.D. degree in molecular biotechnologies from the University of Pisa, Pisa, Italy, in 1999 and 2003, respectively.

She has been a Researcher with the CNR Institute of Clinical Physiology, Pisa, since 2007, where she is involved in DNA-based and protein-based biosensors on the design, synthesis, and purification of bioreceptors and their labelling and immobilization on various kinds of transducers.

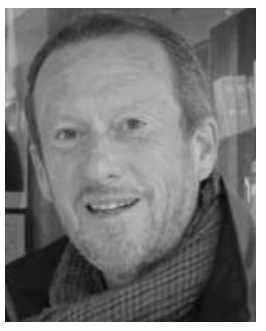

Claudio Domenici received the Laurea degree in physics from the University of Pisa, Pisa, Italy, in 1977, and the Ph.D. degree in material engineering from the Polytechnic of Milan, Milan, Italy, in 1987.

He is currently a Research Director with the CNR Institute of Clinical Physiology, Pisa, with a focus on technological and basic science for medicine. His current research interests include gene-specific biosensors and physicochemical properties of polymeric materials and their interaction with biological systems.

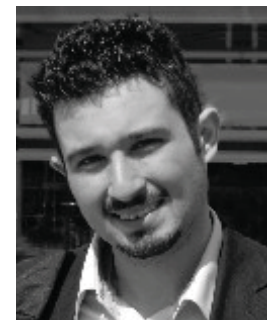

Albert Ruggi received the master's degree in organic chemistry from the University of Rome "La Sapienza," Rome, Italy, in 2006, and the Ph.D. degree from the University of Twente, Enschede, The Netherlands, under the supervision of Prof. Reinhoudt.

He was a Post-Doctoral Researcher with the EPFL, Lausanne, Switzerland. Since 2013, he has been a Maître-Assistant (Lecturer) with the University of Fribourg, Fribourg, Switzerland. His current research interests include the photochemistry of transition metal complexes for imaging and energy-related applications.

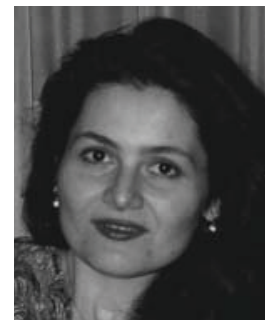

Pisana Placidi received the Ph.D. degree in electronic engineering from the University of Perugia, Perugia, Italy, in 2000.

Since 2005, she has been a Research Assistant with the Department of Electronic and Information Engineering, University of Perugia. Her current research interests include the very large-scale integration design of CMOS active pixel sensors, embedded systems, micromachined gas sensors, interfacing of sensors with programmable system-on-chip, and the design and fabrication of the readout circuits of capacitive biosensors.

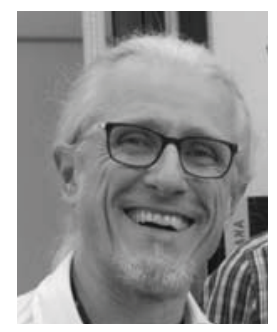

Andrea Scorzoni received the Ph.D. degree in electronic and systems engineering from the University of Bologna, Bologna, Italy, in 1989.

$\mathrm{He}$ has been a Professor of electronics with the Department of Electronic and Information Engineering, University of Perugia, Perugia, Italy, since 1998. His current research interests include electron devices and test structures on siliconand silicon carbide, micromachined gas sensors and solid-state radiation sensors, electromigration, microsystems, embedded systems, radiofrequency identification, brain-computer interfaces, and lab-on-chip.

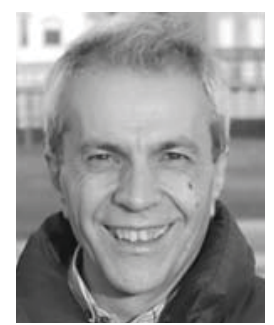

Domenico Caputo is currently an Associate Professor with tenure with the DIET, University of Rome "La Sapienza," Rome, Italy. His current research interests include the development of amorphous silicon photodetectors for detection of radiation from the UV to the near-infrared range and of innovative electronic devices based on amorphous silicon, with a focus on the development of thinfilm photodetector and lab-on-chip device for DNA and micotoxin detection. 